\title{
UN NOUVEL HÔTE D'ESOPHAGOSTOMUM (CONOWEBERIA) BIFURCUM (CREPLIN 1849)
}

\section{Par M. NEVEU-LEMAIRE}

Les collections parasitologiques recueillies en 1926 en Afrique orientale par le professeur G. Lavier, alors membre de la Commission d'étude sur la maladie du sommeil de la Société des Nations, renferment de nombreux exemplaires mâles et femelles d'un œsophagcstome récolté à Entebbe en Uganda, chez un singe mangabey (Cercocebus sp.).

Les formes immatures se trouvaient dans des nodules de la paroi intestinale, dont l'étude anatomo-pathologique a été publiée par G. Lavier et R. Leroux, en 1928.

Les formes adultes occupaient la lumière de l'intestin. Un examen attentif de ces nématodes nous a montré qu'il s'agissait d'CEsophagostomum (Conoweberia) bifurcum (Creplin 1849).

\section{Dimensions}

\begin{tabular}{|c|c|c|}
\hline Esophagostomum (Conoweberia) bifureum & $\sigma^{\circ}$ & \& \\
\hline 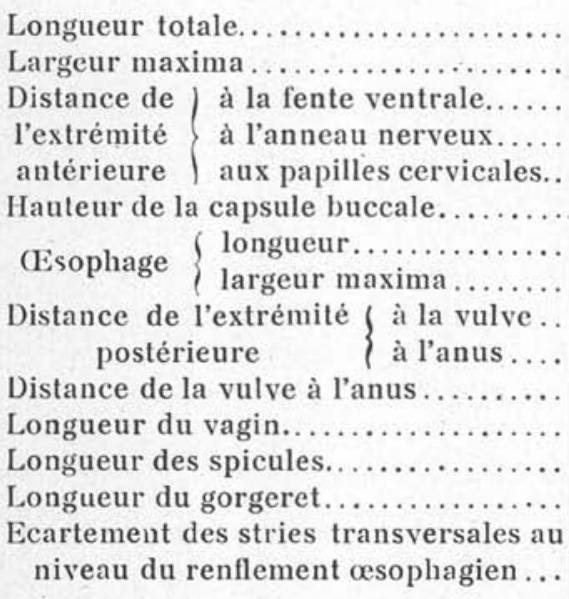 & $\begin{array}{c}9 \text { à } 10 \mathrm{~mm} \text {. } \\
340 \text { à } 370 \mu . \\
200 \text { à } 240 \mu . \\
210 \text { à } 250 \mu \\
320 \mu \\
15 \mu \\
490 \text { à } 540 \mu \\
110 \text { à } 140\end{array}$ & $\begin{array}{c}12 \text { à } 13 \mathrm{~mm} . \\
420 \text { à } 440 \mu \\
220 \text { à } 24 \text { ı } \mu . \\
225 \text { à } 250 \mu \\
280 \mu \\
15 \mu \\
500 \text { à } 540 \mu \\
120 \text { à } 140 \mu \\
400 \text { à } 440 \mu . \\
210 \text { à } 250 \mu \\
190 \mu \\
200 \mu\end{array}$ \\
\hline
\end{tabular}

Annales de Parasitologie, T. XIII, $\mathrm{x}^{\circ} 3 .-1^{\text {er }}$ mai 1935 , p. 203-206. 
Tout récemment, L. Travassos et E. Vogelsang ont fait une étude détaillée de cette espèce ; aussi n'en donnerons-nous pas de nouveau la description et nous bornerons-nous à illustrer cette note de quelques figures et à reproduire les dimensions des exemplaires que nous avons mesurés. Notons en passant qu'aucune des femelles examinées ne contenait d'œufs.

Il résulte, en outre, de l'intéressant travail de Travassos et Vogelsang, qu'un certain nombre d'œsophagostomes, décrits comme espèces distinctes, ne sont, d'après ces auteurs, que l' $E$. bifurcum de Creplin. C'est ainsi qu'ils font tomber en synonymie : $E$. attenuatum $=$ Strongylus attenuatus Leidy 1856, Strongylus cynocephali Molin 1861, CE. stephanostomum Hewlett et Korté 1908, EE. brump$t i$ Railliet et Henry 1905, EEsophagostomum sp., Brumpt 1913, E. apiostomum Leiper 1911 pro parte, Esophagostomum (Conoweberia) sp. Stiles et Hassall 1926 et $C$ E. maurum Hung 1926.

D'après Travassos et Vogelsang, les hôtes d' $E$. bifurcum sont : Cercopithecus (Erythrocebus) patas Schreb., Papio (Choiropithecus) porcarius Bodd., Papio (Choiropithecus) sphinx E. Geoff., Papio (Papio) cynocephalus E. Geoff., Papio (Hamadryas) hamadryas L., Papio (Maimon) maimon L., Macacus (Macacus) rhesus Audebert, Macacus (Cynopithecus) maurus F. Cuv., Anthropopithecus troglodytes L. et Homo sapiens L.

Railliet et Henry rattachent à $G$. brumpti les nématodes incomplètement développés provenant de quelques singes mentionnés cidessus, ainsi que d'un cynocéphale indéterminé, de Papio (Maimon) leucophæus F. Cuv, et de Cynomolgus (Cynomolgus) sinicus L.

Cercopithecus (Cercopithecus) callitrichus E. Geoff. est aussi indiqué parmi les hôtes d'E. bifurcum.

A cette liste déjà longue, nous ajoutons un singe appartenant au genre Cercocebus E. Geoff. 1812. Mais il s'agit de savoir si ce singe, désigné communément sous le nom de « mangabey », est infecté dans la nature. Nous ne saurions l'affirmer, bien que cela soit vraisemblable. Quant à l'individu qui hébergeait les œsophagostomes recueillis par G. Lavier, il vivait en captivité au contact de nombreux cercopithèques et de quelques cynocéphales, hôtes déjà connus de ce nématode, et cela peut suffire à expliquer sa contamination.

Du point de vue de sa distribution géographique, $C E$. bifurcum est surtout un parasite de primates africains. Deux de ses hòtes seulement, Macacus rhesus et Cynomolgus sinicus sont des singes asiatiques. 

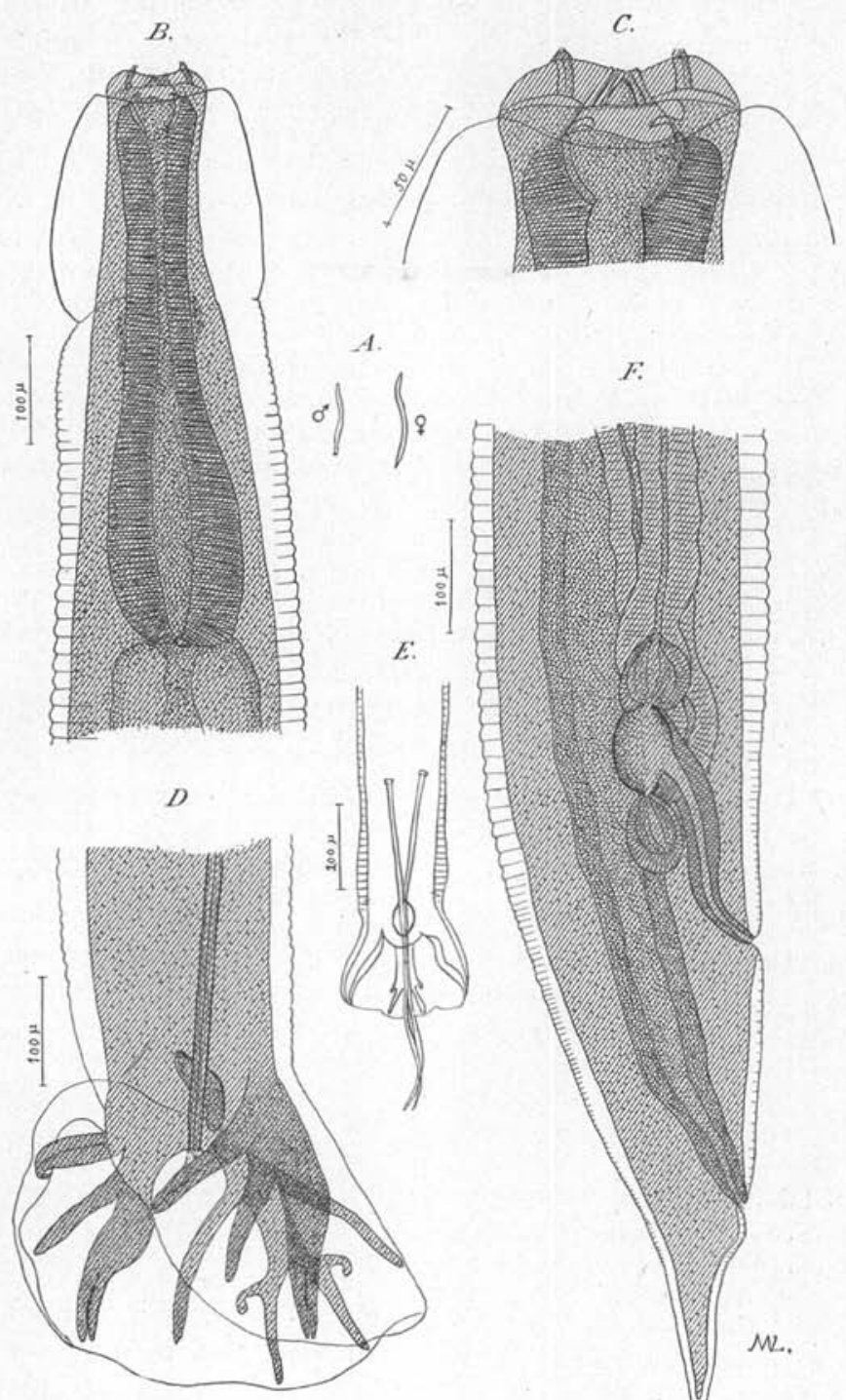

6)

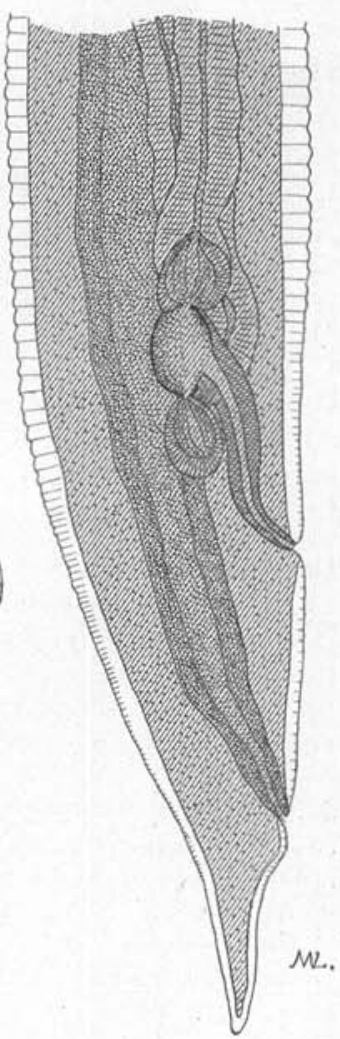

FıG. - Gsophagostomum (Conoweberia) bifurcum (Creplin 1849), - A, mâle et femelle grandeur naturelle; B, extrémité antérieure d'une femelle; C, tête d'une femelle vue à un plus fort grossissement ; D, bourse caudale du mâle; E, extrémité postérieure d'un mâle, spicules et pièce accessoire ; $\mathrm{F}$, extrémité postérieure d'une femelle. 


\section{BIBLIOGRAPHIE}

Brumpt (E.). - Précis de Parasitologie. Masson et Cie, 2॰ éd., Paris, 1913, p. 456 .

Creplin. - Nachträge von Creplin zu Gurlt's Verzeichnisse der Thiere, in welchen Endozoen gefunden worden sind. Arch. f. Naturg., XV, 1849, p. 52 .

Hewlett et KaRTÉ. - On an encysted nematode worm (Esophagostomum stephanostomum) found in the colon of Cynocephalus hamadryas and Macacus rhesus. Journ. of Pathol. and Bacter., XII, 1908, p. 211.

Hung, - Ueber zwei neue parasitische Nematoden, Cooperia fuelleborni, aus dem Wasserbock and Esophagostomum maurum aus dem Mahrenmakak. Arch. f. Sch.-Trop. Hyg., XXX, 1926, p. 421.

Lavier: (G.) et Lenoux (R.). - Lésions intestinales et réactions ganglionnaires par œsophagostomes chez un singe mangabey. Ann. Anat. pathol. et Anat. norm. médico-chirurgicale, $5^{\circ}$ année, $\mathrm{n}^{\circ} 4,1928$.

LEIDY (J.). - A Synopsis of entozoa and some of their ecto-congeners observed by the Author. Proc. acad. of Nat. Sc., Philadelphia, VIII, 1856, p. 42. (Research in Helminth. and Parasit., Smith. misc. coll., XLVI, 1904, p. 84).

LEIPER (R. T.). - The occurrence of Esophagostomum apiostomum as an intestinal parasite of man in Nigeria. Journ. of Trop. Med. and Hyg., XIV, 1911 , p. 116.

Motis. - Il sottordine degli Acrofalli. Mem. Inst. Veneto Sc. Let. e Arti, IX, 1861, p. 427.

Railliet (A.) et HeNry (A.). - Encore un nouveau Sclérostomien (Essophagostomum brumpti nov. sp.) parasite de l'homme. C.R. Soc. biol., LVIII, 1905 , p. 643.

- Sur les Esophagostomes des Primates. C.R. Soc. biol., LX, 1906, p. 448.

- Une seconde espèce d'CEsophagostome parasite de l'homme. Bull. Soc. path. exot., II, 1909, p. 643.

- Les (Esophagostomiens parasites de l'homme. Arch. Parasit., XIV, 1912, p. 562.

Stiles et Hassall. - Key-Catalogue of the worms reported for man. Hyg. Labor., Bull. n ${ }^{\circ}$ 142, 1926.

Stossich (M.). - Sopra alcuni Nematodi. Ann. Museo zool. Univ. Napoli, (N. S.), I, 1904, p. 2.

Thомаs (H. W.). - Esophagostomiasis in Man. Trans. Soc. Trop. Med. and Hyg., III, 1909, p. 44.

Travassos (L.) et Vogelsang (E.). - Contribuição ao conhecimento das especies de Gsophagostomum dos primatas. Mem. Inst. Oswaldo Cruz, XXVI, 1932 , p. 251.

Laboratoire de Parasitologie de la Faculté de médecine de Paris (Directeur : Prof. E. Brumpt). 